Hors-série | 2015

Les élites grecques modernes, $\mathrm{XVIII}{ }^{\mathrm{e}} \mathrm{XX} \mathrm{X}^{\mathrm{e}}$ siècles :

identités, modes d'action, représentations

\title{
La représentation des élites grecques
}

De la collection du portrait peint à celle du portrait photographique

The Representation of Greek Elites: From the Collection of Painted Portrait to

that of the Photographic Portrait

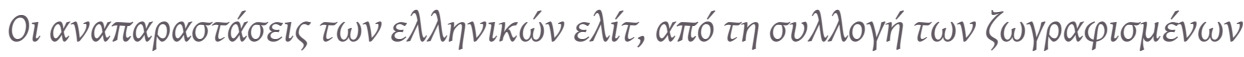

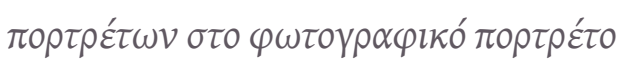

Irini Apostolou

\section{(2) OpenEdition \\ Journals}

Édition électronique

URL : https://journals.openedition.org/ceb/5616

DOI : $10.4000 /$ ceb.5616

ISSN : 2261-4184

Éditeur

INALCO

Édition imprimée

Date de publication : 1 mars 2015

ISBN : 978-2-85831-224-5

ISSN : 0290-7402

Référence électronique

Irini Apostolou, "La représentation des élites grecques », Cahiers balkaniques [En ligne], Hors-série I

2015, mis en ligne le 26 janvier 2016, consulté le 06 juillet 2021. URL : http://journals.openedition.org/ ceb/5616; DOI : https://doi.org/10.4000/ceb.5616

Ce document a été généré automatiquement le 6 juillet 2021.

Cahiers balkaniques est mis à disposition selon les termes de la Licence Creative Commons Attribution - Pas d'Utilisation Commerciale 4.0 International. 


\title{
La représentation des élites grecques
}

\author{
De la collection du portrait peint à celle du portrait photographique \\ The Representation of Greek Elites: From the Collection of Painted Portrait to \\ that of the Photographic Portrait

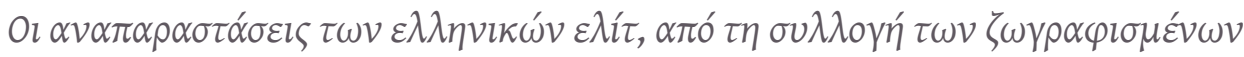

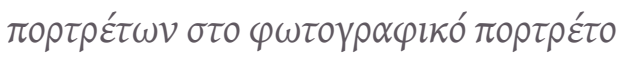

Irini Apostolou

1 Signe de prestige et de respectabilité à l'époque moderne, le portrait peint, initialement réservé à la noblesse et à la haute bourgeoisie, reproduisait les caractéristiques individuelles du modèle et communiquait des informations sur son statut social et sa profession. Associé aux groupes dominants, le portrait officiel s'inscrit dans une longue tradition artistique en France et en Europe. Obéissant à des règles académiques précises, il met en valeur la fonction et la position sociale de l'individu au détriment de sa personnalité intérieure. En outre, son intégration dans des séries des portraits peints ou gravés, appelées galeries de portraits, est révélatrice de l'importance de la représentation picturale à la formation de l'image d'un groupe uni et puissant ${ }^{1}$.

2 La généralisation des portraits photographiques carte de visite d'Eugène Disderi (1854), signala la démocratisation de la représentation de l'individu. Considéré comme moins prestigieux que le portrait peint ou daguerréotype, le portrait photographique fut à l'origine adopté comme mode de représentation surtout par les couches les plus basses de la société française ${ }^{2}$. Le portrait photographique, qui « est une étape dans l'évolution $\mathrm{du}$ portrait en général $»^{3}$, concurrença le portrait peint sans toutefois le faire disparaître. Dans l'espace grec, malgré la réalisation des portraits des Phanariotes et des dignitaires grecs, l'art du portrait resta marginal jusqu'à la formation du nouvel État grec et même durant ses premières décennies. En effet, l'absence d'une bourgeoisie structurée $e^{4}$ et d'institutions artistiques rivalisant avec celles de l'Europe occidentale fut à l'origine d'une production limitée des portraits. Notons également que si la notion de l'élite suppose «non seulement l'idée d'un groupement des membres les plus puissants 
de la société, mais aussi un certain degré de cohésion et une forte conscience de sa propre existence $»^{5}$, ses caractéristiques en Grèce au XIX $x^{e}$ siècle et au début du XXe siècle, n'étaient pas clairement définies. Il fallut attendre la prospérité économique du dernier quart du $\mathrm{xIX}^{\mathrm{e}}$ siècle pour que la bourgeoisie grecque, conformément à son esprit d'européanité, se fit plus systématiquement portraiturer.

Dans le présent article, nous proposons d'étudier la production et l'évolution des séries de portraits peints et photographiques des élites grecques à travers quelques exemples précis mis en parallèle avec des œuvres françaises, qui obéissaient à une longue tradition picturale depuis la Renaissance.

\section{La représentation du pouvoir politique, judiciaire et économique}

4 L'intégration du portrait individuel dans une série contribuait à la mise en valeur soit du lignage familial soit des différentes formes du pouvoir du groupe représenté. Si en France, les monarques, les hommes politiques et les représentants de la nation furent souvent le sujet des galeries de portraits et des recueils gravés afin d'être diffusés auprès du grand public, dans l'espace grec, la première galerie des portraits d'hommes politiques - outre les portraits historiques des héros de la Révolution de Karl Krazeisen - comprend des portraits en pied, des présidents du Sénat ionien, exécutés par Dionysios Végias (1819-1884) selon les règles académiques, qui sont conservés à la salle de réunion du Palais de l'ordre de Saint Michel et Saint Georges ; cette salle est décorée également des effigies de Georges IV, de lord Guilford et de Sir Thomas Maitland, gouverneurs britanniques ainsi que des chevaliers de l'ordre. Parmi les œuvres de la galerie, figure notamment le portrait de Georgios Candianos Romas II, proche de celui de la collection familiale à Zante, qui témoigne de son identité de noble et de son appartenance à l'élite sociale et politique des îles Ioniennes.

5 La perspective idéologique du portrait officiel, qui essayait d'effacer «les traits spécifiques qui pourraient nuire au message à transmettre $»^{6}$, fut souvent abordée par les chercheurs. En Europe occidentale et tout particulièrement en France, le mécanisme de la mise en scène du pouvoir royal favorisa la réalisation du portrait du roi, dont le symbolisme fut à l'origine de la longue tradition du portrait officiel du président de la République française. Depuis Adolphe Thiers, les présidents se font portraiturer au début de leur mandat pour leur portrait officiel qui, accroché dans les mairies et largement diffusé sous forme de copies, affirme le prestige de leur fonction et symbolise leur présence dans l'espace.

6 À l'opposé des présidents et des hommes politiques français, l'élite politique grecque ne fut pas systématiquement représentée, quoique des portraits officiels des rois Othon et Georges $\mathrm{I}^{\mathrm{er}}$ en costume royal ou militaire aient été exécutés et inclus dans des séries de peintures. Leur présence ainsi que celle de leurs épouses et d'autres membres de la famille royale dans des collections des différentes institutions comme celles de l'Université d'Athènes et de la Société des amis de l'Instruction (hétérie "philecpedeutique ») rappelaient l'appui du pouvoir royal. Lors de l'exil de la reine Amalia, qui fut «sa protectrice ", la Société s'adressa à la reine Olga, qui, à son tour, accepta d'assumer le même rôle. Offerts à la Société par l'ambassadeur de la Russie en 
Grèce, son portrait et celui de Georges $\mathrm{I}^{\text {er }}$, exécutés par Spyridon Prossalentis en 1871, furent exposés dans un endroit spécial lors des célébrations du Cinquantenaire du royaume de Grèce auxquelles le couple royal, l'héritier et la princesse Alexandra assistèrent. Reconnaissante du soutien royal, la Société chargea Nicéphore Lytras de réaliser les portraits du roi Othon et de la reine Amalia, qu'il représenta dans un cadre montrant les antiquités d'Athènes. Dans le même esprit, la Banque de Grèce voulut également rendre hommage au roi Othon et en même temps légitimer sa fondation au service de l'État ${ }^{7}$. Reprenant son portrait réalisé pour la Société des amis de l'Instruction, Lytras ${ }^{8} \mathrm{y}$ ajouta l'acte constitutif de la fondation de la Banque du 30 mars 1841, qui symbolisa la protection du pouvoir royal et son intérêt pour le développement économique du pays.

7 À l'instar du roi, les hommes politiques se faisaient également représenter quoique leurs portraits, comme ceux de Charilaos Trikoupis ${ }^{9}$ (qui de tendance libérale fut plusieurs fois Premier ministre), fussent en général des commandes privées et non pas publiques. En effet, plusieurs portraits des premiers présidents de la Chambre des députés comme celui de Thrassyvoulos Zaïmis (1820-1862), réalisé par Dionysios Tsokos, qui ornent actuellement le foyer et la librairie du Parlement grec, furent exécutés longtemps après leur disparition ${ }^{10}$. Si Nicolaos Orloff reconstitua en 1930 un portrait collectif des hommes politiques dans une Séance du Parlement, leurs portraits furent collectionnés progressivement, comme en attestent celui de Georgios Sissinis (1769-1831) réalisé par Chronis Botsoglou (né en 1941), qui reprit un ancien portrait, et celui de Nicolaos Levidis (1848-1942), député et plusieurs fois ministre, peint par Ernestos Carter (1924-1992), qui malgré ses tendances modernistes, réalisa une œuvre conforme aux conventions du portrait officiel.

8 À l'instar du pouvoir législatif et exécutif dont les portraits étaient pliés à « l'impératif de la ressemblance, de la fidélité au particulier, au détail, à l'effet de réel $\aleph^{11}$, le pouvoir judiciaire se servit du portrait pour affirmer son prestige. En France, des portraits officiels des présidents et des membres du Parlement datant du XVII ${ }^{\mathrm{e}}$ et du XVIII ${ }^{\mathrm{e}}$ siècle ainsi que des juges et des procureurs généraux ornent souvent les salles des hauts tribunaux comme dans le cas de la Cour de cassation française, qui conserve plusieurs portraits comme celui d'Alexis Ballot-Beaupré (1836-1917), réalisé apparemment d'après une photographie par J. P. Valéry.

9 Quoique beaucoup plus modestes que les portraits des magistrats français, ceux des présidents, des vice-présidents et des procureurs, qui sont actuellement conservés à la salle d'assemblée plénière de la Cour de cassation de Grèce, témoignent de la même volonté de leur rendre hommage. En effet, sous le règne de George $\mathrm{I}^{\mathrm{er}}$, juges, avocats et professeurs de droit connurent une ascension sociale, qui facilita souvent leur entrée en politique. Il fallut néanmoins attendre le début du $\mathrm{xIX}^{\mathrm{e}}$ siècle pour que Georges Iakovidis exécutât le portrait d'Anastassios Polyzoïdes (1802-1873), correspondant à la troisième assemblée grecque (1826-1827) et connu pour avoir refusé de signer la condamnation de Colocotronis. De plus, il réalisa celui de Christodoulos Clonaris (1788-1849), premier président, principal inspirateur de l'œuvre législative élaborée par le gouvernement de Capodistrias et professeur émérite de droit à l'Université d'Athènes dont un portrait est également conservé dans la collection de Rizareios $^{12}$. Souvent médiocrement exécutées, les représentations officielles des magistrats ainsi que de celles des avocats du barreau d'Athènes furent néanmoins une trace matérielle et visuelle de leur appartenance à des corps d'élite. 
10 En Grèce, la constitution des élites fut liée à la croissance de l'économie et au développement des activités commerciales de l'État. La création de la Banque Nationale de Grèce en 1841, qui mena une action sociale et culturelle importante, marqua l'économie grecque durant les $\mathrm{XIX}^{\mathrm{e}}$ et $\mathrm{XX}^{\mathrm{e}}$ siècles. La commande par Marcos Renieris des bustes de Georgios Stavrou, premier gouverneur de la Banque, à Ioannis Kossos et de Paul Calligas à Georgios Vroutos fut à l'origine de la constitution de la collection des œuvres d'art commémoratives des gouverneurs de la Banque Nationale de Grèce, dont les portraits furent confiés à des artistes réputés comme Nicéphore Lytras qui, dans une approche à la fois réaliste et moderne, représenta Calligas (1896), Marcos Renieris (1897) et Georgios Stavrou assis, sans aucun effort de les rajeunir. De même, Georges Iacovidis portraitura Stéphanos Streit (1835-1920), Alexandros Zaïmis (1855-1936) et Ioannis Valaoritis (1911-1914) adaptant son style personnel aux conventions $\mathrm{du}$ portrait officiel. Archétypes de la bourgeoisie capitaliste, les gouverneurs de la Banque Nationale de Grèce appartenaient également à l'élite intellectuelle du pays, c'est pourquoi des portraits de Renieris et de Streit par Iacovidis figurent également dans la collection de l'Université d'Athènes. Notons que, même après la généralisation du portrait photographique et l'enregistrement par la photographie du fonctionnement de la Banque Nationale de Grèce, la tradition de l'exécution des portraits peints de ses gouverneurs par des artistes réputés, qui perdure jusqu'au Xxi ${ }^{\mathrm{e}}$ siècle ${ }^{13}$, est révélatrice de l'utilisation du portrait peint comme moyen de mettre en valeur sa fonction et de montrer son appartenance à un corps d'élite.

\section{Les galeries de portraits des institutions éducatives}

11 La constitution des galeries de portraits servait également à l'affirmation du prestige des institutions à travers la représentation de leurs membres éminents. En France, des universités, des bibliothèques, des académies et des hôpitaux constituaient des séries de portraits de leurs bienfaiteurs et de leurs professeurs afin de leur rendre hommage et de commémorer leur œuvre, qui était étroitement liée à leur réputation institutionnelle. Depuis le $\mathrm{XVIII}^{\mathrm{e}}$ siècle, l'université de Montpellier réunit progressivement une riche collection de 230 portraits peints et de bustes sculptés des professeurs de médecine et de quelques chirurgiens dont celui de François Gigot de Lapeyronie par Hyacinthe Rigaud. Son initiative fut suivie par la Faculté des sciences de la ville, dont le conseil d'administration, adoptant la proposition du doyen Joseph Diez Gergonne, décida en 1836 l'exécution d'une galerie des portraits de ses professeurs, qui devaient se faire représenter au cours de leur première année de leurs fonctions ${ }^{14}$.

12 L'impressionnante collection de 347 portraits, réalisés par 90 artistes de l'Université $\mathrm{d}^{\prime}$ Athènes ${ }^{15}$ fut également un élément essentiel pour la préservation de sa mémoire historique et pour l'affirmation de son prestige. Classés en cinq catégories, ils représentent les maîtres de la nation, les fondateurs, les bienfaiteurs et donateurs, les personnalités de l'histoire grecque et les hommes politiques ainsi que les professeurs de l'établissement ${ }^{16}$. Peints systématiquement dès la première décennie de l'université jusqu'au début des années 1960, ils étaient en général des commandes directes du conseil du rectorat aux artistes ${ }^{17}$. Parmi les portraits de la collection, qui comprend des œuvres d'une qualité artistique inégale ${ }^{18}$, figurent également des tableaux exécutés par des portraitistes réputés comme Nicéphore Lytras qui représenta Théodoros Aretaios 
(1829-1893), professeur de chirurgie et bienfaiteur, et Georges Iakovidis qui immortalisa Démosthène Tsivanopoulos (1838-1921). Exécutés en général après leur disparition, les portraits, dont certains étaient médiocrement peints, s'appuyaient souvent sur des portraits photographiques ${ }^{19}$.

Quoique moins importante, la collection des portraits de l'École nationale polytechnique (Metsovio) possède des caractéristiques communes avec celle de l'Université nationale d'Athènes. Outre les portraits de ses bienfaiteurs, Nicolaos Stournaras, Georges Averoff et Michaël Tossitsas et de sa donatrice Sophia Chlorou, détruits lors des événements de novembre 1991, on y trouve ceux de ses directeurs comme Friedrich von Zentner (1837-1841) et Angelos Ghinis (1910-1920 et 1923-1927), dont l'exécution par Georges Iakovidis fut financée par le legs Averoff. De plus, nous y trouvons plusieurs portraits de professeurs, qui furent exécutés essentiellement au $\mathrm{Xx}^{\mathrm{e}}$ siècle par des artistes célèbres comme Giannis Moralis, peintre du portrait d'Emmanouil Kriezis (1880-1967). La constitution progressive de la collection et l'intérêt porté actuellement à sa restauration et à la commande de nouveaux portraits à la place de ceux qui furent endommagés lors des événements de novembre 1991, témoignent actuellement de la volonté de l'institution de mettre en valeur sa mémoire historique et de rendre hommage aux personnages engagés dans son histoire.

14 Plus tardive, mais obéissant au même esprit de commémoration de l'histoire de l'institution, la collection artistique de l'Université de Thessalonique-Aristote, comprend soixante-seize portraits des professeurs, qui ont été exécutés par des artistes réputés dont certains étaient originaires de Thessalonique. À l'exception du portrait d'Alexandros Papanastassiou, exécuté par Constantinos Parthenis, les portraits, peints après la disparition des professeurs étaient commandés par le conseil du rectorat après des rapports du conseil artistique. S'appuyant sur des clichés photographiques afin de rendre fidèlement leur physionomie ${ }^{20}$, les peintres mettaient en avant le rôle social, moral et professionnel du modèle.

institutions d'enseignement supérieur, des fondations éducatives comme la Fondation Rizareios (fondée en 1841) et la Société des amis de l'Instruction (fondée en 1836) se constituèrent des galeries de portraits peints pour honorer leurs donateurs, directeurs et professeurs. Leur commande fut un moyen d'exprimer leur gratitude aux personnes, qui avaient contribué à leur développement et en même temps de montrer leur protection par l'élite politique, économique et judiciaire.

Dans l'idée de rendre hommage à son fondateur Georgios Rizaris, la fondation Rizareios commanda son portrait à Georges Iakovidis, qui le représenta en pied, tenant son acte fondateur, et ayant comme fond le rocher de l'Acropole ${ }^{21}$. Le portrait du donateur Efthymios Christodoulou, également par Iakovidis, et d'autres médiocrement peints représentant surtout des hommes d'Église et/ou ayant participé aux Lumières grecques - dont certains comme Neophytos Vamvas et Anthimos Gazis figurent dans la collection de l'Université d'Athènes - contribuèrent au rehaussement du prestige de la fondation.

La Société des amis de l'Instruction d'Athènes, dont le développement et la prospérité furent intimement liés à son administration par les figures éminentes de l'élite grecque, décida dès que ses finances lui permirent de commander les portraits de Ioannis Kokkonis (1796-1864), de Georgios Koundouriotis, d'A. Metaxas et de Leontios Robotis ${ }^{22}$. Outre les commandes de la société, la collection s'enrichit des portraits offerts par des membres de la famille des présidents défunts. Ainsi le portrait 
d'Alexandros Mavrokordatos lui fut offert par son fils Nicolaos tandis que celui de Léon Melas fut donné par son frère. Parmi les portraits des présidents de la Société figurent des personnalités éminentes de la société grecque que nous retrouvons également dans des galeries d'autres institutions. Ainsi Constantinos Simandiras, président de la Cour de cassation (1891-1911) et de la Société des amis de l'Instruction (1895-1899), fut également représenté par Spyridon Vikatos pour la collection de l'Université d'Athènes. De facture traditionnelle, les portraits peints des présidents de la Société des amis de l'instruction d'Athènes, dont la tradition persiste jusqu'aujourd'hui, mirent en valeur leur œuvre et contribuèrent à la réputation de l'école ${ }^{23}$.

\section{Le portrait photographique diffusé, collectionné et échangé}

L'image de Georges $\mathrm{I}^{\mathrm{er}}$, photographié régulièrement à Athènes par Petros Moraïtis ${ }^{24}$, portraitiste officiel du roi et puis de la famille royale, et, à Paris, par Solon Vathis, fut reproduite dans la presse illustrée française de l'époque (l'Illustration, le Petit Journal, le Journal illustré). Vathis remit notamment au Gaulois « le portrait du roi Georges de Grèce et ceux de MM. Tricoupis et Boudouris, le premier président du conseil démissionnaire, et le second président de la Chambre hellène $»^{25}$. Outre l'exécution des portraits photographiques officiels du roi, la reine olga constitua une importante collection de portraits de la famille royale, plus intimes que les habituels portraits protocolaires ${ }^{26}$. Lors de leur séjour à Paris, la famille royale posa à Solon Vathis comme nous en rapporte le Gaulois de $1886^{27}$ :

Nous sommes allés, hier, sur l'invitation de M. Solon Vathis, photographe, rue Vivienne, 43, visiter les salons et les ateliers de pose de l'artiste grec. Nous avons vu chez lui de superbes photographies. Ce sont celles de la famille royale, qui, pendant son séjour à Paris, est allée plusieurs fois chez M. Solon Vathis. Un groupe représentant

S.A.R. le duc de Sparte et S.A.R. le prince André est remarquablement réussi.

Les hommes politiques, les intellectuels et les bourgeois grecs se faisaient également photographier chez Moraïtis et les autres photographes de la capitale ${ }^{28}$. Depuis l'âge classique et même avant, le portrait, qui «soutient la mémoire en ravivant l'image qu'elle conserve $"{ }^{29}$, fut souvent échangé entre amants, amis et correspondants permettant ainsi la reconnaissance de leur physionomie avant d'être diffusé par la presse au large public. Collectionnées et réunies dans des albums, leurs photographies témoignaient également du milieu et des affinités de leur possesseur. Les lettres de l'historien et intellectuel Antonio Rubio échangées avec ses correspondants grecs fournissent des informations intéressantes sur la fonction des portraits photographiques. Dimitrios Kambouroglou lui écrivit notamment qu'il lui ferait «le plus grand plaisir et honneur s'il lui [était] facile de lui envoyer sa photographie » en ajoutant qu'il ferait de même dans sa prochaine lettre ${ }^{30}$. De son côté, après avoir remercié Spyridon Lambros de lui avoir envoyé sa photo, Rubio lui écrivit qu'il s'empressait de lui envoyer la sienne prise deux ans auparavant ${ }^{31}$. En outre, il louait à Dimitrios Vikélas, qu'il connaissait déjà par une photographie fournie par Sagnier ${ }^{32}$, son portrait photographique réalisé par Vathis ${ }^{33}$ : 
Je vous dois mil remerciements par votre magnifique photographie, qui est bien supérieure de la première de vous que je connaissais, et qui fait honneur au photographe Grec M. Solon Vathis.

En plus de l'utilisation des clichés photographiques pour la réalisation des portraits posthumes, l'adoption de la photographie comme mode de représentation officielle à coût réduit mit à l'épreuve le portrait peint dans la constitution de galeries des portraits comme celle des maires de Thessalonique ou celle des ministres de l'Éducation et des affaires religieuses nommés après la transition démocratique (Métapoliteusis), dont les portraits réunis sur un panneau, rappellent leur physionomie et marquent la continuité de leur fonction dans le temps.

Le professeur Louis Bruntz, directeur supérieur de l'École de pharmacie, après avoir demandé l'exposition dans la salle du conseil des portraits photographiques, prit la décision de créer, assisté par l'Association des anciens élèves et du conseil de l'École, une galerie photographique des portraits des anciens professeurs de l'École de Strasbourg, et de celle de Nancy ${ }^{34}$.

En Grèce, parallèlement à l'exécution des portraits officiels par les peintres, dont certains s'appuyaient sur des clichés photographiques, les photographes immortalisèrent les membres de la direction et les professeurs et captèrent la vie universitaire. Les archives photographiques de l'Université d'Athènes conservent des portraits individuels et de groupe des professeurs et d'étudiants, souvent montrés dans des situations ordinaires du travail à un moment donné et dans des conditions précises. Ces clichés, dont certains, comme celui montrant Xavier Landerer, professeur de pharmacologie entouré de ses assistants, présentent souvent une qualité esthétique puisque « le photo-portrait est à la fois célébration du sujet - un art de la personne - et genre artistique - un art de l'image $»^{35}$, sont des témoignages matériels du passé historique de l'institution. À l'occasion des célébrations du Centenaire de l'Université, les ouvrages retraçant l'histoire des différentes facultés de l'institution furent illustrés d'une galerie des portraits représentant leurs professeurs. De plus, la publication commémorative, qui s'ouvrait sur les portraits d'Othon $\mathrm{I}^{\mathrm{er}}$, de Georges II, du premier et du centième recteur et du ministre de l'Éducation, reproduisit, outre les portraits des professeurs de l'établissement depuis sa fondation et de ses évergètes, des photographies de groupe montrant le comité des célébrations, le conseil des finances ainsi que les festivités et des moments de la vie universitaire ${ }^{36}$.

De même, l'École nationale polytechnique conserve un important fonds photographique lié aux activités photographiques de ses professeurs comme Philippos Margaritis (1810-1892) ${ }^{37}$.

Outre les portraits photographiques individuels, très codés par ailleurs, le fonds comprend des portraits commémoratifs des groupes de professeurs et d'étudiants pris souvent à la fin de l'année scolaire ou lors de l'enseignement. Plusieurs clichés montrant les étudiants, parfois accompagnés de leurs professeurs lors des excursions scientifiques et les diplômés de l'École polytechnique témoignent des liens de groupe ainsi que du rôle de l'institution dans leur carrière professionnelle.

Si l'acte photographique est une coupe dans le temps et l'espace ${ }^{38}$, les clichés photographiques isolent des moments représentatifs du quotidien de la vie

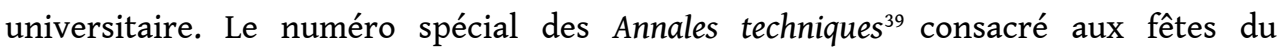
centenaire de l'École polytechnique, reproduisit, outre les portraits peints des bienfaiteurs, directeurs, recteurs et professeurs, plusieurs portraits photographiques 
de groupe. Notons également qu'en général, il s'agit d'images le plus souvent narratives et banalisées, qui montrent la cohésion de ses membres.

De même, les portraits photographiques des instituteurs de la Société des amis de l'Instruction et les clichés représentant les élèves en classe, en train de faire de la gymnastique et de participer aux fêtes de la fin de l'année scolaire, dont certaines illustrent les ouvrages commémoratifs publiés à l'occasion du cinquantenaire et du centenaire de la Société, mettent en avant les instituteurs et les étudiants comme collectif contribuant à la réputation de l'école ${ }^{40}$.

\section{Conclusion}

La mise en valeur des institutions grecques se concrétisa également par la constitution de séries de portraits. Témoignages des personnalités éminentes de la société grecque, les portraits des membres de l'élite politique, judiciaire, financière et intellectuelle, qui étaient compris le plus souvent dans des séries des peintures, malgré la généralisation $\mathrm{du}$ portrait photographique, participaient à l'enregistrement de leur mémoire de groupe et affirmaient leur pouvoir. Témoignage de l'attachement des institutions à leur passé, les représentations individuelles et collectives des membres de l'élite grecque contribuaient à sa cohésion. Rappel visuel également des personnes, qui contribuèrent à l'administration, à la prospérité et à l'œuvre éducative et scientifique des différents établissements, les portraits peints et photographiques célébrèrent leur histoire à travers l'image de leurs acteurs.

\section{Bibliographie}

AYENSA I PRAT Eusebi, 2006, Epistolari grec Antoni Rubió $i$ Lluch ; correspondència recollida $i$ anotada per, anys 1880-1888, Barcelona : Institut d'Estudis catalans.

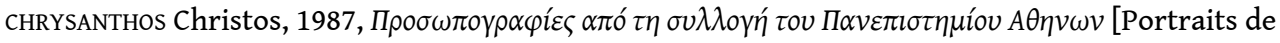
la collection de l'Université nationale d'Athènes 1837-1987], Athènes : Université nationale d'Athènes.

CONSTANTINOU Fani, TSIRGialou Aliki (dir.), 2004, Athens 1839-1900. A photographic record, Athènes : Benaki Museum.

COQUERY Emmanuel et alii, 1997, Visages du Grand Siècle : le portrait français sous le règne de Louis XIV, 1660-1715 [exposition, Nantes, Musée des beaux-arts, 20 juin-15 septembre 1997, Toulouse, Musée des Augustins, 8 octobre 1997-5 janvier 1998]/[catalogue par Paris : Somogy ; Nantes : Musée des beaux-arts ; Toulouse : Musée des Augustins].

DUBoIs Philippe, 1990, l'Acte photographique, Paris : Nathan Université. 
DULIEU Louis, 1996, « les Portraits des universitaires montpelliérains à travers les galeries de tableaux », Bulletin de l'Académie des sciences et lettres de Montpellier, nouvelle série, tome 27, p. 301-310.

DWAN Renato, 2000, « Un outil puissant : les théories de l'élite et l'étude de la construction européenne », in Élisabeth du RÉAU (dir.), Europe des élites ? Europe des peuples ? La construction de l'espace européen, Paris : Presses Sorbonne Nouvelle.

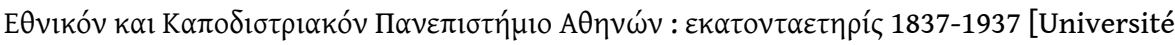
nationale et capodistrienne d'Athènes : le centenaire 1837-1937], 1937, Athènes : Pyrsos.

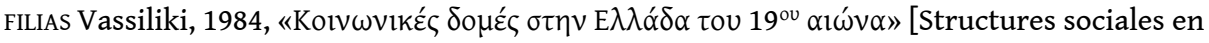

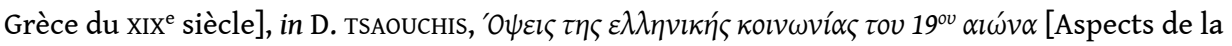
société grecque au XIX ${ }^{\mathrm{e}}$ siècle], Athènes : Estia.

FREUND Gisèle, 1936, la Photographie en France au dix-neuvième siècle : essai de sociologie et d'esthétique, Paris : A. Monnier.

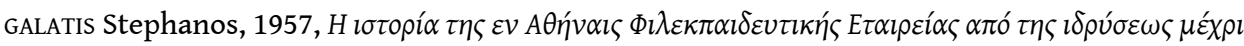

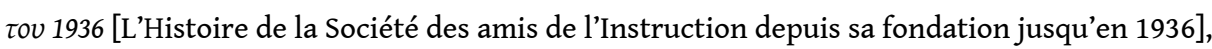
Athènes, (tapuscrit consulté à la Bibliothèque Gennadius).

ITZHAK Goldberg, 1999, « Le visage abstrait », in François AUBRAL, Dominique CHÂTEAU (dir.), Figure, Figurai, L’Harmattan : Paris.

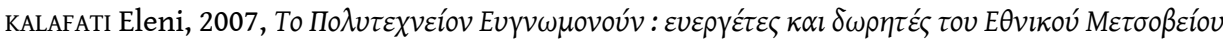

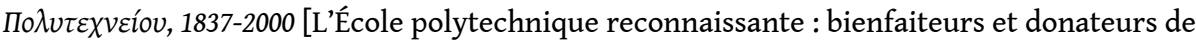
l'École polytechnique 1837-2000 revu], Athènes : École polytechnique nationale.

LABRUDE Pierre, 1996, « Les professeurs strasbourgeois de la galerie des portraits de la Faculté de pharmacie de Nancy ", La Revue de l'Histoire de la pharmacie, n 308, p. 39-52.

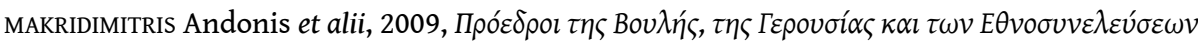
1821-2008 [Présidents du Parlement grec, du Sénat et des Assemblées nationales 1821-2008], Athènes : Fondation du Parlement hellénique pour le parlementarisme et la démocratie.

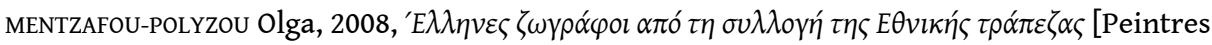
grecs de la collection de la Banque Nationale], Pinacothèque nationale, Musée Alexandros Soutzos, Banque Nationale de Grèce, Athènes : Banque Nationale.

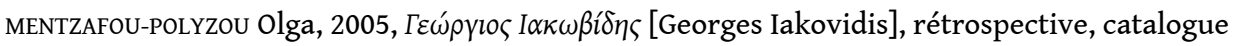
d'exposition, Musée Alexandros Soutzos : [Athènes].

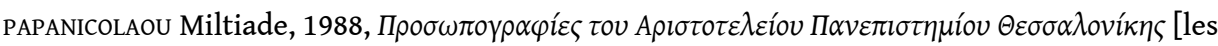
Portraits de l'Université Aristote de Thessalonique], Thessalonique : Université Aristote.

POLLEROSS Frédéric, 2010, « La galerie de Portraits entre architecture et littérature : essai de typologie ", in Claire CONSTANS, Mathieu DA VINHA (dir.), 2010, les Grandes galeries européennes XVII ${ }^{e}$ XIX ${ }^{e}$ siècles, Centre de recherche du château de Versailles : Éditions de la Maison des sciences de l'homme, p. 67-90.

ROUILLÉ André, 1982, l'Empire de la photographie : photographie et pouvoir bourgeois, 1839-1870, Paris : Le Sycomore.

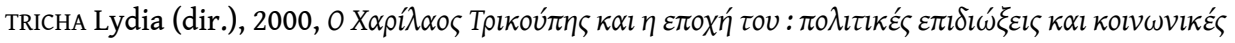
$\sigma v v \theta \eta ́ \kappa \varepsilon \varsigma$ [Charilaos Tricoupis et son époque : préoccupations politiques et conditions sociales], Athènes : Papazissi. 
VIGIÉ Muriel, (2000), le Portrait officiel en France du ve au XXe siècle, Paris : FVM.

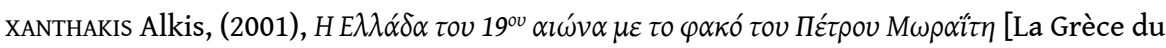
$\mathrm{XIX}{ }^{\mathrm{e}}$ siècle à travers l'objectif de Petros Moraïtis], Athènes : Potamos.

\section{Presse}

Le Gaulois du 30 octobre 1886.

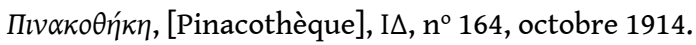

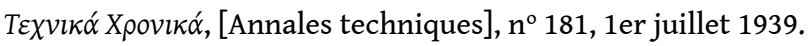

\section{Sitographie}

Thierry GRILLET, « Petite phénoménologie du portrait photographique », http:// expositions.bnf.fr/portraits/arret/1/index.htm.

\section{NOTES}

1. Friedrich POLLERoss, «La galerie de portraits entre architecture et littérature : essai de typologie", dans les Grandes galeries européennes XVII ${ }^{e}$-XIXe siècles, Claire consTANS, Mathieu DA VINHA (dir.), Centre de recherche du château de Versailles : Éditions de la Maison des sciences de l'homme, 2010, p. 67-90.

2. André RouilLÉ, l'Empire de la photographie : photographie et pouvoir bourgeois, 1839-1870, Paris : le Sycomore, 1982, p. 62.

3. Gisèle FREUND, la Photographie en France au dix-neuvième siècle: essai de sociologie et d'esthétique, Paris : A. Monnier, 1936, p. 11.

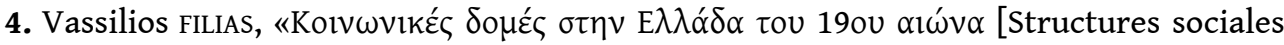
en Grèce du xIX siècle]» in Dimitrios TSAOUchIS, 'O $\alpha \iota \omega ́ v \alpha »$ [Aspects de la société grecque au XIX ${ }^{e}$ siècle], Athènes : Estia, 1984, p. 16.

5. Renata DWAN, « Un outil puissant : les théories de l'élite et l'étude de la construction européenne ", dans É. du RÉAU, (dir.), Europe des élites ? Europe des peuples ? La construction de l'espace européen, Paris: Presses Sorbonne Nouvelle, 2000, Coll. Espace européen, p. 27.

6. Muriel vigiÉ, Le Portrait officiel en France du $V^{e}$ au XXe siècle, Paris : FVM, 2000, p. 8.

7. Sur le cadre idéologique de la constitution de la collection artistique de la Banque

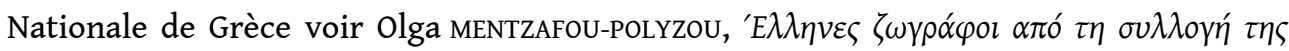

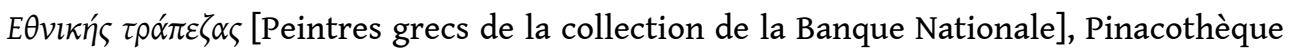
nationale, Musée Alexandros Soutzos, Banque Nationale de Grèce, [Athènes] : Banque Nationale, 2008, p. 15-26.

8. Ibid., $\mathrm{n}^{\circ} 88$, p. 53 .

9. Plusieurs portraits peints et photographiques de Tricoupis sont reproduits dans

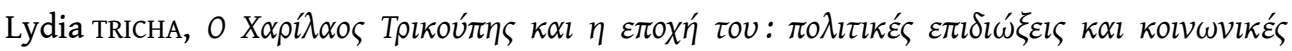


$\sigma v v \theta \eta ́ k \varepsilon \varsigma$ [Charilaos Tricoupis et son époque : préoccupations politiques et conditions sociales], Athènes : éd. Papazissi, 2000.

10. La collection du parlement comprend 56 portraits de premiers ministres et 65 portraits des résidents du Parlement, du Sénat et des assemblées générales (information fournie par Théodoros Koutsogiannis), dont certains illustrent l'ouvrage

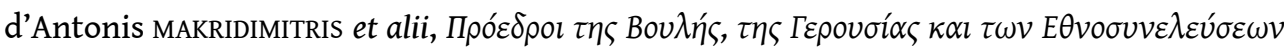
1821-2008, [Présidents du Parlement grec, du Sénat et des Assemblées nationales 1821-2008], Athènes : Fondation du Parlement hellénique pour le parlementarisme et la démocratie, 2009.

11. Itzhak GOLDBERG, «Le visage abstrait» in Figure, Figurai, François AUBRAL, Dominique CHÂTEAU (dir.), Paris : L'Harmattan, 1999, p. 125.

12. Commandés par le ministère de la Justice les deux portraits furent accrochés en grande pompe dans la salle d'assemblée plénière en 1914. Sur ce sujet, consulter

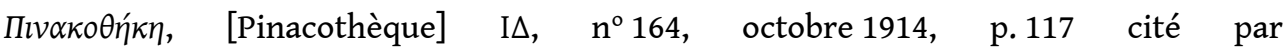

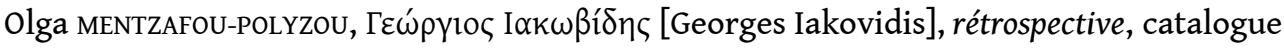
d'exposition, Musée Alexandros Soutzos, [Athènes], 2005, p. 241.

13. Les portraits des gouverneurs sont reproduits dans Olga MENTZAFOU-POLYZOU, op. cit., 2008, p. 27-45.

14. Louis DULIEU, «Les portraits des universitaires montpelliérains à travers les galeries de tableaux ", Bulletin de l'Académie des sciences et lettres de Montpellier, 1996, nouvelle série, tome 27, p. 301-310.

15. Pour plus d'informations sur la collection de l'Université d'Athènes et pour une

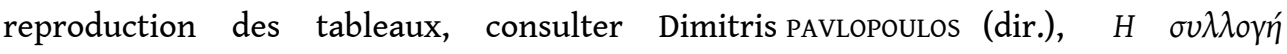

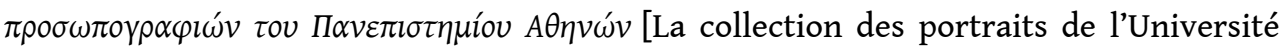
d'Athènes], Athènes : Université nationale d'Athènes, 2009.

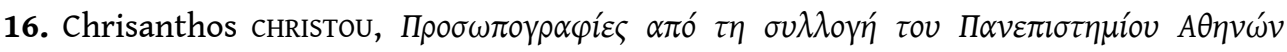
[Portraits de la collection de l'Université nationale d'Athènes 1837-1987], Athènes : Université nationale d'Athènes, 1987, p. 12.

17. Sur ce sujet voir PAVLoPoulos, op. cit., notice 12 p. 87.

18. CHRISTOU, op. cit. p. 24.

19. PAVLOPOULOS, op. cit., p. 88.

20. Sur les portraits datant essentiellement après 1945, voir Miltiadis PAPANICOLAOU,

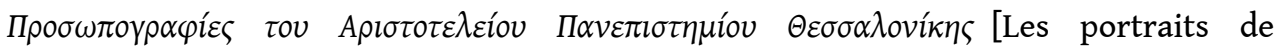
l'Université Aristote de Thessalonique], Thessalonique: Université Aristote, 1988, et spécialement les pages 24, 29-30.

21. Olga MENTZAFOU-POLYZOU, op. cit., 2005, p. 162

22. Pour plus d'informations sur la commande des tableaux des présidents consulter

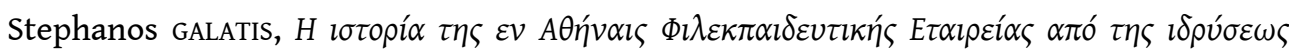

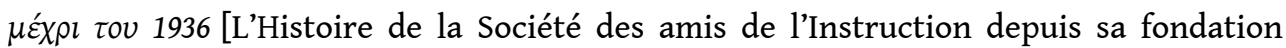
jusqu'en 1936], Athènes, 1957 (tapuscrit consulté à la Bibliothèque Gennadius).

23. La collection des portraits de la Société des amis de l'Instruction est reproduite dans

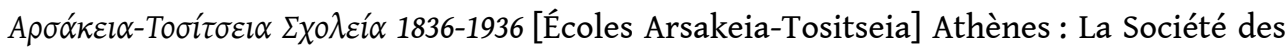
amis de l'Instruction, 1996, p. 368-371. 


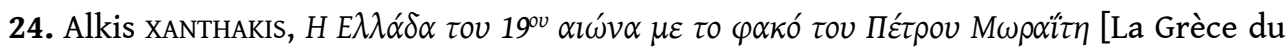
XIX ${ }^{\mathrm{e}}$ siècle à travers l'objectif de Petros Moraïtis], Athènes : Potamos, 2001.

25. Le Gaulois du 27 janvier 1895, p. 3.

26. XANTHAKIS, op. cit., p. 82-83, 134-151.

27. Le Gaulois du 30 octobre 1886, p. 2.

28. Voir notamment les portraits photographiques des hommes politiques reproduits dans Fani constantinou, Aliki tsirgialou (dir.), Athens 1839-1900. A photographic record, Athènes: Benaki Museum, 2004, pp. 114-129.

29. Visages du Grand Siècle: le portrait français sous le règne de Louis XIV, 1660-1715, [exposition, Nantes, Musée des beaux-arts, 20 juin-15 septembre 1997, Toulouse, Musée des Augustins, 8 octobre 1997-5 janvier 1998]/[catalogue par Emmanuel coQUERY, Olivier BONFAIT, Dominique BRÊME, et alii.] Paris : Somogy ; Nantes : Musée des beauxarts ; Toulouse : Musée des Augustins, 1997, p. 22.

30. Athènes, « lettre du 16 juillet 1884 », Eusebi AYENSA I PRAT, Epistolari grec Antoni Rubió $i$ Lluch; correspondència recollida $i$ anotada per anys 1880-1888, Barcelona : Institut d'Estudis catalans, 2006, vol. I, p. 256.

31. Barcelone, « lettre du 5 avril $1882 »$, ibidem., p. 136.

32. Barcelone, « lettre du 26 novembre 1883 », ibid., p. 237.

33. Barcelone, « lettre du 10 février $1887 »$, ibid., p. 306.

34. Pierre LABRUDE, «Les professeurs strasbourgeois de la galerie des portraits de la Faculté de pharmacie de Nancy ", La revue de l'Histoire de la pharmacie, n 308, 1996, p. 39-52.

35. Thierry GRILLET, « Petite phénoménologie du portrait photographique ", http:// expositions.bnf.fr/portraits/arret/1/, consulté le 8 octobre 2015.

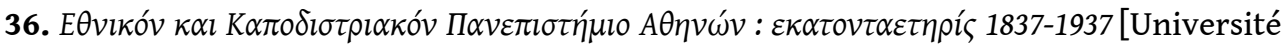
nationale et capodistrienne d'Athènes : le centenaire 1837-1937], Athènes : Pyrsos, 1937.

37. Pour une reproduction des portraits et pour une histoire des collections, consulter

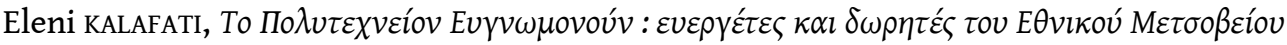

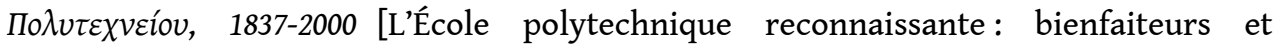
donateurs de l'École polytechnique 1837-2000 revu], Athènes, École polytechnique nationale, 2007.

38. Philippe Dubois, l'Acte photographique, Paris : Nathan Université, 1990, chap. 4.

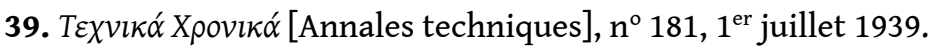

40. Des clichés du fonds photographique de la Société des amis de l'Instruction et de

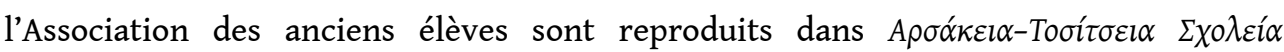
1836-1936 [Écoles Arsakeia-Tositseia] Athènes : La Société des amis de l'Instruction, 1996. 


\section{RÉSUMÉS}

Dans l'espace grec, malgré la réalisation des portraits des Phanariotes et des dignitaires, l'art du portrait resta marginal jusqu'à la formation du nouvel État grec et durant ses premières décennies. En effet, l'absence d'une bourgeoisie structurée et d'institutions artistiques fut à l'origine d'une production limitée des portraits. Dans le présent article, nous proposons d'étudier, à travers quelques exemples précis mis en parallèle avec des œuvres françaises, la production et l'évolution des séries de portraits peints et photographiques des élites grecques qui obéissaient à une longue tradition picturale depuis la Renaissance.

In the Greek area, despite the realization of portraits of Phanariots and dignitaries, the art of portraiture remained marginal till the creation of the new Greek State and during its first decades. Indeed the lack of a structured bourgeoisie and of arts institutions was a source of a limited production of portraits. In the present paper, we want to study the production and evolution of the sets of painted and photographic portraits of the Greek elites through some precise examples in parallel with French works, obeying a long pictorial tradition since the Renaissance.

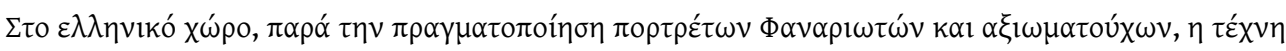

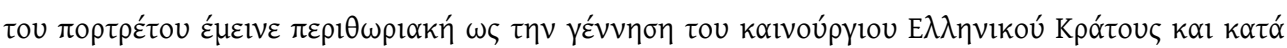

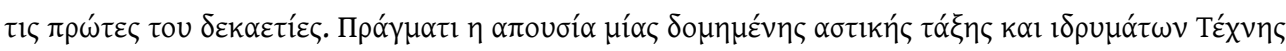

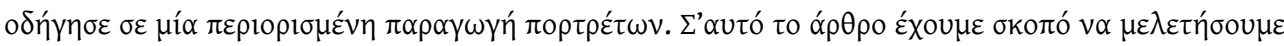

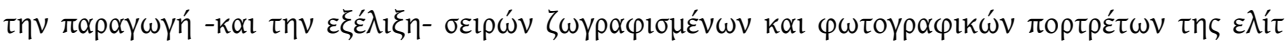

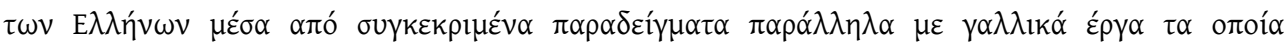

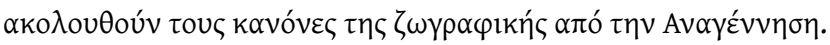

\section{INDEX}

Index géographique : Grèce

Keywords : Portrait, Photography, Greece, Nineteenth century, Twentieth century, Art, Painting motsclesmk ПОРТРЕТ, ФОТОГРАФИЈА, ГРЦИЈА, ДЕВЕТНАЕСЕТТИОТ ВЕК, ДВАЕСЕТТИОТ ВЕК, УМЕТНОСТ, СЛИКАРСТВО

motsclestr Portre, Fotoğrafçlık, Yunanistan, Ondokuzuncu yüzyıl, Yirminci yüzyıl, Sanat, Resim

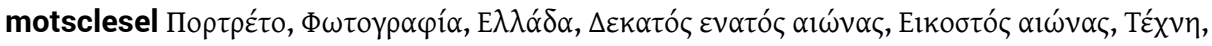

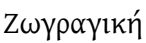

Mots-clés : photographie, portrait

Index chronologique : vingtième siècle, dix-neuvième siècle

\section{AUTEUR}

\section{IRINI APOSTOLOU}

Université nationale d'Athènes 